NONCOMMUTATIVE GEOMETRY

AND QUANTUM GROUPS

BANACH CENTER PUBLICATIONS, VOLUME 61

INSTITUTE OF MATHEMATICS

POLISH ACADEMY OF SCIENCES

WARSZAWA 2003

\title{
THE STRUCTURE OF CORINGS WITH A GROUPLIKE ELEMENT
}

\author{
TOMASZ BRZEZIŃSKI \\ Department of Mathematics, University of Wales Swansea \\ Singleton Park, Swansea SA2 8PP, U.K. \\ E-mail: T.Brzezinski@swansea.ac.uk,URL:http://www-maths.swan.ac.uk/staff/tb
}

\begin{abstract}
Characteristic properties of corings with a grouplike element are analysed. Associated differential graded rings are studied. A correspondence between categories of comodules and flat connections is established. A generalisation of the Cuntz-Quillen theorem relating existence of connections in a module to projectivity of this module is proven.
\end{abstract}

1. Introduction. The notion of a coring was introduced by Sweedler in [27] as a generalisation of coalgebras over commutative rings to the case of non-commutative rings. While the theory of coalgebras has been developed quite substantially over the last quarter of the century, most notably after the discovery of large classes of examples coming from quantum groups, the progress in the coring theory was hampered by the lack of examples. Recently M. Takeuchi realised that the compatibility condition between an algebra and a coalgebra known as an entwining [8] can be recast in terms of a coring. Entwining structures and the associated categories of entwined modules [4] unify and generalise various categories of Hopf modules such as relative Hopf modules [28], [14], Yetter-Drinfeld modules [31], [24] or Doi-Koppinen Hopf modules [15], [21]. Several classes of examples of entwining structures are known (cf. recent monograph [9]). Thus it turns out that the theory of corings is very rich in examples. Once a coring is built from an entwining structure, the category of entwined modules is isomorphic to the category of comodules of this coring. Various properties of entwined modules can be understood and more simply derived from general properties of corings and their comodules. A programme of studying corings from this point of view was initiated in [6] and is now gaining momentum with several new results reported in the area (cf. [1], [7], [16], [17], [29], [30]).

The present paper is devoted to the study of general properties of those corings which have a grouplike element. These are corings most closely related to the original example

2000 Mathematics Subject Classification: 16W30, 13B02.

The paper is in final form and no version of it will be published elsewhere. 
studied by Sweedler in [27], and they reveal the richness of structure, with vistas on the (noncommutative) descent theory and noncommutative geometry. We begin in Section 2 by recalling the basic definitions and examples, and by deriving elementary properties of corings with a grouplike element. We then proceed in Section 3 to recall their connection with graded differential rings, and reveal their close relationship with noncommutative connections in Section 4.

All rings in this paper are associative and unital, and the unit of a ring $R$ is denoted by $1_{R}$, while $\mathbf{M}_{R}$ denotes the category of right $R$-modules, and ${ }_{R} \mathbf{M}$ the left $R$-modules etc. All algebras and coalgebras are over a commutative ring $k$, undecorated tensor products and homomorphisms are over $k$. The identity morphism for any object $X$ in any category is denoted by the same symbol $X$.

2. Basic properties. Given a ring $R$, an $(R, R)$-bimodule $\mathfrak{C}$ is called an $R$-coring if there exist $(R, R)$-bimodule maps $\Delta_{\mathfrak{C}}: \mathfrak{C} \rightarrow \mathfrak{C} \otimes_{R} \mathfrak{C}$ and $\epsilon_{\mathfrak{C}}: \mathfrak{C} \rightarrow R$, such that

$$
\left(\Delta_{\mathfrak{C}} \otimes_{R} \mathfrak{C}\right) \circ \Delta_{\mathfrak{C}}=\left(\mathfrak{C} \otimes_{R} \Delta_{\mathfrak{C}}\right) \circ \Delta_{\mathfrak{C}}, \quad\left(\epsilon_{\mathfrak{C}} \otimes_{R} \mathfrak{C}\right) \circ \Delta_{\mathfrak{C}}=\left(\mathfrak{C} \otimes_{R} \epsilon_{\mathfrak{C}}\right) \circ \Delta_{\mathfrak{C}}=\mathfrak{C} .
$$

The map $\Delta_{\mathfrak{C}}$ is called the coproduct while the map $\epsilon_{\mathfrak{C}}$ is called the counit of $\mathfrak{C}$. A morphism of $R$-corings $\mathfrak{C}$ and $\mathfrak{D}$ is an $(R, R)$-bimodule map $\mathfrak{f}: \mathfrak{C} \rightarrow \mathfrak{D}$ such that $\Delta_{\mathfrak{D}} \circ \mathfrak{f}=\left(\mathfrak{f} \otimes_{R} \mathfrak{f}\right) \circ \Delta_{\mathfrak{C}}$ and $\epsilon_{\mathfrak{D}} \circ \mathfrak{f}=\epsilon_{\mathfrak{C}}$. Given an $R$-coring $\mathfrak{C}$, a right $\mathfrak{C}$-comodule is a right $R$-module $\mathfrak{M}$ together with a right $R$-module map $\rho^{\mathfrak{M}}: \mathfrak{M} \rightarrow \mathfrak{M} \otimes_{R} \mathfrak{C}$ such that

$$
\left(\rho^{\mathfrak{M}} \otimes_{R} \mathfrak{C}\right) \circ \rho^{\mathfrak{M}}=\left(\mathfrak{M} \otimes_{R} \Delta_{\mathfrak{C}}\right) \circ \rho^{\mathfrak{M}}, \quad\left(\mathfrak{M} \otimes_{R} \epsilon_{\mathfrak{C}}\right) \circ \rho^{\mathfrak{M}}=\mathfrak{M} .
$$

A map between right $\mathfrak{C}$-comodules is a right $R$-module map respecting the coactions, i.e., $\mathfrak{f}: \mathfrak{M} \rightarrow \mathfrak{N}$ satisfies $\rho^{\mathfrak{N}} \circ \mathfrak{f}=\left(\mathfrak{f} \otimes_{R} \mathfrak{C}\right) \circ \rho^{\mathfrak{M}}$. The category of right $\mathfrak{C}$-comodules is denoted by $\mathbf{M}_{R}^{\mathfrak{C}}$, and their morphisms by $\operatorname{Hom}_{R}^{\mathfrak{C}}(-,-)$. The actions of $\Delta_{\mathfrak{C}}$ and $\rho^{\mathfrak{M}}$ on elements are denoted by the Sweedler notation $\Delta_{\mathfrak{C}}(c)=c_{(1)} \otimes_{R} c_{(2)}, \rho^{\mathfrak{M}}(m)=m_{(0)} \otimes_{R} m_{(1)}$. Similarly one defines left $\mathfrak{C}$-comodules, and bicomodules.

Any ring $R$ can be viewed as an $R$-coring via the identity maps. In this case the category of comodules is the same as the category of right $R$-modules. Furthermore, given a ring extension $S \rightarrow R$, the $(R, R)$-bimodule $\mathfrak{C}=R \otimes_{S} R$ is an $R$-coring with the coproduct $\Delta_{\mathfrak{C}}\left(r \otimes_{S} r^{\prime}\right)=r \otimes_{S} 1_{R} \otimes_{S} r^{\prime}$ and the counit $\epsilon_{\mathfrak{C}}\left(r \otimes_{s} r^{\prime}\right)=r r^{\prime}$, for all $r, r^{\prime} \in R$. We refer to this coring as the canonical Sweedler coring associated to a ring extension $S \rightarrow R$. The category of $\mathfrak{C}$-comodules in this case is isomorphic to the category of (noncommutative) descent data [20], [11] for the ring extension $S \rightarrow R$. Finally, let $(A, C)_{\psi}$ be an entwining structure over a commutative ring $k$, i.e., $A$ is a $k$-algebra, $C$ is a $k$-coalgebra with a counit $\epsilon$ and coproduct $\Delta$, and $\psi: C \otimes A \rightarrow$ $A \otimes C$ is a $k$-linear map satisfying mixed distributive law conditions (cf. [8] or [6] for details). Then $\mathfrak{C}=A \otimes C$ is an $A$-coring with the following structure. Left $A$ multiplication is given by the product in $A$, right $A$-multiplication is provided by the map $\psi$, i.e., $(a \otimes c) a^{\prime}=a \psi\left(c \otimes a^{\prime}\right)$, the coproduct and the counit are $\Delta_{\mathfrak{C}}=A \otimes$ $\Delta$ and $\epsilon_{\mathfrak{C}}=A \otimes \epsilon$. In this case $\mathbf{M}_{A}=\mathbf{M}_{A}^{C}(\psi)$, the latter denoting the category of $(A, C)_{\psi}$-entwined modules, i.e., right $A$-modules and $C$-comodules $M$ with a compatibility condition $\rho^{M}(m a)=m_{(0)} \psi\left(m_{(1)} \otimes a\right)$, for all $m \in M, a \in A$, introduced in $[4]$. 
A non-zero element $g$ of an $R$-coring $\mathfrak{C}$ is said to be a grouplike element if $\Delta_{\mathfrak{C}}(g)=$ $g \otimes_{R} g$ and $\epsilon_{\mathfrak{C}}(g)=1_{R}$. A ring $R$ viewed as a trivial $R$-coring has a grouplike element $1_{R}$. Similarly, the canonical Sweedler coring associated to $S \rightarrow R$ has a grouplike element $1_{R} \otimes_{S} 1_{R}$. In general the existence of a grouplike element determines when a ring is itself a comodule of a coring (cf. [6, Lemma 5.1]). Precisely, an $R$-coring $\mathfrak{C}$ has a grouplike element $g$ if and only if $R$ is a right $\mathfrak{C}$-comodule. The coaction reads $\rho^{R}(r)=g r$. For example, in the case of an entwining structure $(A, C)_{\psi}$, the corresponding coring has a grouplike element if and only if $A$ is an entwined module. In this case $g=\rho^{A}\left(1_{A}\right) \in A \otimes C$, where $\rho^{A}$ is a coaction of $C$ on $A$.

Given an $R$-coring with a grouplike element $g$ and a right $\mathfrak{C}$-comodule $\mathfrak{M}$ with a coaction $\rho^{\mathfrak{M}}: \mathfrak{M} \rightarrow \mathfrak{M} \otimes_{R} \mathfrak{C}$, one defines $g$-coinvariants of $\mathfrak{M}$ as an Abelian group

$$
\mathfrak{M}_{g}^{c o \mathfrak{C}}=\left\{m \in \mathfrak{M} \mid \rho^{\mathfrak{M}}(m)=m \otimes_{R} g\right\} .
$$

The $g$-coinvariants $S$ of $R$ form a subring of $R$ equal to the centraliser of $g$ in $R$, i.e., $S=\{s \in R \mid s g=g s\}$.

Given any right $S$-module $M$, where $S$ are $g$-coinvariants of $R$, one can consider $M \otimes_{S} R$ as a right $\mathfrak{C}$-comodule via the coaction

$$
\rho^{M \otimes_{S} R}: M \otimes_{S} R \rightarrow M \otimes_{S} R \otimes_{R} \mathfrak{C} \cong M \otimes_{S} \mathfrak{C}, \quad m \otimes_{S} r \mapsto m \otimes_{S} g r .
$$

The assignment $M \mapsto M \otimes_{S} R$ defines a covariant functor $-\otimes_{S} R: \mathbf{M}_{S} \rightarrow \mathbf{M}_{R}^{\mathfrak{C}}$, known as an induction functor. If $f: M \rightarrow N$ is a morphism in $\mathbf{M}_{S}$ then $f \otimes_{S} R: m \otimes_{S} r \mapsto$ $f(m) \otimes_{S} r$. Note that $f \otimes_{S} R$ is a morphism in $\mathbf{M}_{R}^{\mathfrak{C}}$ since $\rho^{N \otimes_{S} R}\left(f(m) \otimes_{S} r\right)=f(m) \otimes_{S} g r$ and $\left(f \otimes_{S} R \otimes_{R} \mathfrak{C}\right) \circ \rho^{M \otimes_{S} R}\left(m \otimes_{S} r\right)=\left(f \otimes_{R} \mathfrak{C}\right)\left(m \otimes_{R} g r\right)=f(m) \otimes_{S} g r$. In the opposite direction [6, Proposition 5.2], the assignment $G_{g}: \mathbf{M}_{R}^{\mathfrak{C}} \rightarrow \mathbf{M}_{S}, \mathfrak{M} \mapsto \mathfrak{M}_{g}^{\text {coc }}$, defines a covariant functor, known as a $g$-coinvariants functor, which is the right adjoint of the induction functor $-\otimes_{S} R: \mathbf{M}_{S} \rightarrow \mathbf{M}_{R}^{\mathfrak{C}}$. On morphisms $G_{g}$ acts as a restriction of the domain, i.e., for any $\mathfrak{f}: \mathfrak{M} \rightarrow \mathfrak{N}$ in $\mathbf{M}_{R}^{\mathfrak{C}}, G_{g}(\mathfrak{f})=\left.\mathfrak{f}\right|_{\mathfrak{M}_{g}^{\text {cocec }}}$.

EXAMPLE 2.1. Let $\mathfrak{C}$ be an $R$-coring with a grouplike element $g$, and let $S=R_{g}^{\text {coc }}$ be the subring of $g$-coinvariants. View $\mathfrak{C}$ as a right or left $\mathfrak{C}$-comodule via the coproduct $\Delta_{\mathfrak{C}}$. Then $R \cong \mathfrak{C}_{g}^{c o \mathfrak{C}}$ as $(R, S)$-bimodules.

Proof. First note that since $\Delta_{\mathfrak{C}}$ is an $(R, R)$-bimodule map, the set of $g$-coinvariants $\mathfrak{C}_{g}^{c o \mathfrak{C}}=\left\{c \in \mathfrak{C} \mid \Delta_{\mathfrak{C}}(c)=c \otimes_{R} g\right\}$ is a left $R$-module. Thus $\mathfrak{C}_{g}^{c o \mathfrak{C}}$ is an $(R, S)$-bimodule. Consider a left $R$-module map $\phi: R \rightarrow \mathfrak{C}_{g}^{c o c}, r \mapsto r g$. Since $S$ centralises $g$ in $R$, we have for all $s \in S$ and $r \in R, \phi(r s)=r s g=r g s=\phi(r) s$, i.e., $\phi$ is an $(R, S)$-bimodule map. It is well-defined since $\Delta_{\mathfrak{C}}(r g)=r g \otimes_{R} g$, i.e., $r g \in \mathfrak{C}_{g}^{c o \mathfrak{C}}$ for all $r \in R$, as needed. Now consider $\phi^{-1}=\left.\epsilon_{\mathfrak{C}}\right|_{\mathfrak{C}_{g}^{c o \mathfrak{C}}}: \mathfrak{C}_{g}^{c o \mathfrak{C}} \rightarrow R$. Clearly $\phi^{-1}$ is an $(R, S)$-bimodule map (since $\epsilon_{\mathfrak{C}}$ is an $(R, S)$-bimodule map). Furthermore for all $r \in R, \phi^{-1} \circ \phi(r)=\epsilon_{\mathfrak{C}}(r g)=r$. Notice that if $c \in \mathfrak{C}_{g}^{c o \mathfrak{C}}$, then $\Delta_{\mathfrak{C}}(c)=c \otimes_{R} g$, hence applying $\epsilon_{\mathfrak{C}} \otimes_{R} \mathfrak{C}$ we have $c=\epsilon_{\mathfrak{C}}(c) g$. Using this fact take any $c \in \mathfrak{C}_{g}^{c o \mathfrak{C}}$ and compute $\phi \circ \phi^{-1}(c)=\phi\left(\epsilon_{\mathfrak{C}}(c)\right)=\epsilon_{\mathfrak{C}}(c) g=c$. This proves that $\phi^{-1}$ is the inverse of $\phi$ in ${ }_{R} \mathbf{M}_{S}$, i.e., $R \cong \mathfrak{C}_{g}^{c o \mathfrak{C}}$ as $(R, S)$-bimodules.

The $g$-coinvariants functor can be viewed as a hom-functor.

Proposition 2.2. Let $\mathfrak{C}$ be an $R$-coring with a grouplike element $g$ and let $S=R_{g}^{\text {coc }}$ be the subring of $g$-coinvariants. For any $\mathfrak{M} \in \mathbf{M}_{R}^{\mathfrak{C}}$ view $\operatorname{Hom}_{R}^{\mathfrak{C}}(R, \mathfrak{M})$ as a right $S$-module 
via $(\mathfrak{f} s)(r)=\mathfrak{f}(s r)$, for all $\mathfrak{f} \in \operatorname{Hom}_{R}^{\mathfrak{C}}(R, \mathfrak{M}), r \in R$ and $s \in S$. Then $\operatorname{Hom}_{R}^{\mathfrak{C}}(R, \mathfrak{M}) \cong \mathfrak{M}_{g}^{\text {coC }}$ as right $S$-modules.

Proof. First note that $\operatorname{Hom}_{R}^{\mathfrak{C}}(R, \mathfrak{M})$ is a right $S$-module as stated. Indeed, for all $s \in S, r \in R$ and $\mathfrak{f} \in \operatorname{Hom}_{R}^{\mathfrak{C}}(R, \mathfrak{M})$ one computes

$$
\begin{aligned}
(\mathfrak{f} s)\left(r_{(0)}\right) \otimes_{R} r_{(1)} & =(\mathfrak{f} s)\left(1_{R}\right) \otimes_{R} g r=\mathfrak{f}(s) \otimes_{R} g r=\mathfrak{f}\left(1_{R}\right) \otimes_{R} s g r \\
& =\mathfrak{f}\left(1_{R}\right) \otimes_{R} g s r=\mathfrak{f}\left((s r)_{(0)}\right) \otimes_{R}(s r)_{(1)} \\
& =\rho^{\mathfrak{M}}(\mathfrak{f}(s r))=\rho^{\mathfrak{M}}((\mathfrak{f} s)(r)),
\end{aligned}
$$

i.e., $\mathfrak{f} s$ is a right $\mathfrak{C}$-comodule map as required. Clearly the multiplication $\mathfrak{f} s$ is associative. Now to any $\mathfrak{f} \in \operatorname{Hom}_{R}^{\mathfrak{C}}(R, \mathfrak{M})$ assign $m_{\mathfrak{f}}=\mathfrak{f}\left(1_{R}\right) \in \mathfrak{M}$. Since $\mathfrak{f}$ is a right $\mathfrak{C}$-comodule map we have

$$
\rho^{\mathfrak{M}}\left(m_{\mathfrak{f}}\right)=\rho^{\mathfrak{M}}\left(\mathfrak{f}\left(1_{R}\right)\right)=\mathfrak{f}\left(1_{(0)}\right) \otimes_{R} 1_{(1)}=\mathfrak{f}\left(1_{R}\right) \otimes_{R} g=m_{\mathfrak{f}} \otimes_{R} g,
$$

so that $m_{\mathfrak{f}} \in \mathfrak{M}_{g}^{c o \mathfrak{C}}$. Furthermore for all $s \in S$ we have $m_{\mathfrak{f} s}=(\mathfrak{f} s)\left(1_{R}\right)=\mathfrak{f}(s)=$ $\mathfrak{f}\left(1_{R}\right) s=m_{\mathfrak{f}} s$. All this means that the assignment $\mathfrak{f} \mapsto m_{\mathfrak{f}}$ is a right $S$-module morphism $\operatorname{Hom}_{R}^{\mathfrak{C}}(R, \mathfrak{M}) \rightarrow \mathfrak{M}_{g}^{c o \mathfrak{C}}$.

Conversely to any $m \in \mathfrak{M}_{g}^{c o \mathfrak{C}}$ assign $\mathfrak{f}_{m} \in \operatorname{Hom}_{R}(R, \mathfrak{M}), \mathfrak{f}_{m}: r \mapsto m r$. Since $m$ is in $g$-coinvariants, and $\rho^{\mathfrak{M}}$ is a right $R$-module morphism one can take any $r \in R$ and compute

$$
\rho^{\mathfrak{M}}\left(\mathfrak{f}_{m}(r)\right)=\rho^{\mathfrak{M}}(m r)=m \otimes_{R} g r=\mathfrak{f}_{m}\left(1_{R}\right) \otimes_{R} g r=\mathfrak{f}_{m}\left(r_{(0)}\right) \otimes_{R} r_{(1)},
$$

i.e., $\mathfrak{f}_{m}$ is a right $\mathfrak{C}$-comodule map. Since the assignments $\mathfrak{f} \mapsto m_{\mathfrak{f}}$ and $m \mapsto \mathfrak{f}_{m}$ described above are restrictions of the natural isomorphism $\operatorname{Hom}_{R}(R, \mathfrak{M}) \cong \mathfrak{M}$, they are inverses to each other as required.

The existence of a grouplike element $g$ in $\mathfrak{C}$ allows for a direct sum decomposition of $\mathfrak{C}, \mathfrak{C} \cong R \oplus \operatorname{ker} \epsilon_{\mathfrak{C}}$ as $(S, R)$-bimodules and $(R, S)$-bimodules, where $S=R_{g}^{c o \mathfrak{C}}$. The $(S, R)$-bimodule isomorphism is given by $u_{R}: c \mapsto\left(\epsilon_{\mathfrak{C}}(c), g \epsilon_{\mathfrak{C}}(c)-c\right)$, with the inverse $u_{R}^{-1}(r, c)=g r-c$, while the $(R, S)$-bimodule isomorphism is $u_{L}: c \mapsto\left(\epsilon_{\mathfrak{C}}(c), c-\epsilon_{\mathfrak{C}}(c) g\right)$, with the inverse $u_{L}^{-1}(r, c)=c+r g$. Since $\operatorname{ker} \epsilon_{\mathfrak{C}}$ is an $(R, R)$-bimodule there is a natural ring structure on $R \oplus$ ker $\epsilon_{\mathfrak{C}}$ with the product given by $(r, c)\left(r^{\prime}, c^{\prime}\right)=\left(r r^{\prime}, r c^{\prime}+c r^{\prime}\right)$, for all $r, r^{\prime} \in R$ and $c, c^{\prime} \in \operatorname{ker} \epsilon_{\mathfrak{C}}$, and the unit $(1,0)$. Using the isomorphisms $u_{L}$ or $u_{R}$ this ring structure can be pulled back to $\mathfrak{C}$, and the product comes out as

$$
c c^{\prime}=\epsilon_{\mathfrak{C}}(c) c^{\prime}+c \epsilon_{\mathfrak{C}}\left(c^{\prime}\right)-\epsilon_{\mathfrak{C}}(c) g \epsilon_{\mathfrak{C}}\left(c^{\prime}\right),
$$

for all $c, c^{\prime} \in \mathfrak{C}$, and the unit is $g$. Furthermore the counit $\epsilon_{\mathfrak{C}}: \mathfrak{C} \rightarrow R$ is a ring map split by ring maps $i_{L}, i_{R}: R \rightarrow \mathfrak{C}$ given by, $i_{L}: r \mapsto r g, i_{R}: r \mapsto g r$. In the case of the canonical Sweedler coring, the above ring structure appears in [23, Section 1.2] in the context of a braiding related to the noncommutative descent theory.

Another basic property of corings with a grouplike element is the fact that their dual rings are augmentation rings. Recall from [27] that the left dual of $\mathfrak{C},{ }^{*} \mathfrak{C}={ }_{R} \operatorname{Hom}(\mathfrak{C}, R)$ is a unital ring with unit $\epsilon_{\mathfrak{C}}$ and the product defined for all $\xi, \xi^{\prime} \in{ }^{*} \mathfrak{C}$ and $c \in \mathfrak{C}$ by $\left(\xi \xi^{\prime}\right)(c)=\xi\left(c_{(1)} \xi^{\prime}\left(c_{(2)}\right)\right)$. ${ }^{*} \mathfrak{C}$ is an $(R, R)$-bimodule via $\left(r \xi r^{\prime}\right)(c)=\xi(c r) r^{\prime}$. In the case of the canonical coring associated to a ring extension $S \rightarrow R,{ }^{*} \mathfrak{C}$ is simply the left $S$ endomorphism ring of $R$, while in the case of the $A$-coring $\mathfrak{C}=A \otimes C$ associated to an en- 
twining structure $(A, C)_{\psi},{ }^{*} \mathfrak{C}$ is a $\psi$-twisted convolution product algebra $\left(\operatorname{Hom}(C, A), *_{\psi}\right)$ introduced in [5] (see [22] for a special case). In general, every right $\mathfrak{C}$-comodule $\mathfrak{M}$ is a left ${ }^{*} \mathfrak{C}$-module via $\xi m=m_{(0)} \xi\left(m_{(1)}\right)$. Recently it has been shown in [30] that $\mathbf{M}_{R}^{\mathfrak{C}}$ is a full subcategory of $* \mathfrak{C} \mathbf{M}$ if and only if $\mathfrak{C}$ is a locally projective left $R$-module (cf. [32] for the definition of a locally projective module). Next recall from [10, p. 143] that a ring $R$ is called a left augmentation ring if there exists an $R$-module $M$ and a left $R$-module morphism $\pi: R \rightarrow M$. $M$ is called an augmentation module.

Proposition 2.3. Let $\mathfrak{C}$ be an $R$-coring with a grouplike element $g$. Then ${ }^{*} \mathfrak{C}$ is a left augmentation ring with the augmentation module $R$. The left action of $* \mathfrak{C}$ on $R$ is provided by $\xi r=\xi(g r)$ for all $r \in R, \xi \in *^{*} \mathfrak{C}$.

Proof. That the map given above defines a left action of $* \mathfrak{C}$ on $R$ follows from the fact that $R$ is a right $\mathfrak{C}$-comodule, and hence it is a left ${ }^{*} \mathfrak{C}$-module as recalled above. The augmentation $\pi:{ }^{*} \mathfrak{C} \rightarrow R$ is given by $\xi \mapsto \xi(g)$. To show that $\pi$ is a left ${ }^{*} \mathfrak{C}$-module map, take any $\xi, \xi^{\prime} \in{ }^{*} \mathfrak{C}$ and compute: $\pi\left(\xi \xi^{\prime}\right)=\left(\xi \xi^{\prime}\right)(g)=\xi\left(g \xi^{\prime}(g)\right)=\xi\left(g \pi\left(\xi^{\prime}\right)\right)=\xi \pi\left(\xi^{\prime}\right)$.

Proposition 2.3 extends an observation made in [19] in the case of finitely generated corings.

3. The Amitsur complex. There is a close relationship between corings with a grouplike element and a certain type of differential graded rings, first described by Rojter [25].

First recall that a differential graded ring is an $\mathbb{N} \cup\{0\}$-graded ring $\Omega=\bigoplus_{n=0}^{\infty} \Omega^{n}$ together with an additive degree one operation $d: \Omega^{\bullet} \rightarrow \Omega^{\bullet+1}$ such that $d \circ d=0$, which satisfies the graded Leibniz rule, i.e., for all elements $\omega^{\prime} \in \Omega$ and all degree $n$ elements $\omega$, $d\left(\omega \omega^{\prime}\right)=d(\omega) \omega^{\prime}+(-1)^{n} \omega d\left(\omega^{\prime}\right)$.

Given a coring with a grouplike element one constructs an associated differential graded ring as follows [25]. Consider a tensor ring $\Omega(\mathfrak{C})=\bigoplus_{n=0}^{\infty} \Omega^{n}(\mathfrak{C})$, where $\Omega^{0}(\mathfrak{C})=R$ and $\Omega^{n}(\mathfrak{C})=\mathfrak{C} \otimes_{R} \mathfrak{C} \otimes_{R} \cdots \otimes_{R} \mathfrak{C}$ (n-times). Define a degree one additive map $d: \Omega(\mathfrak{C}) \rightarrow$ $\Omega(\mathfrak{C})$ via $d(r)=g r-r g$, and

$$
\begin{aligned}
d\left(c^{1} \otimes_{R} \cdots \otimes_{R} c^{n}\right)= & g \otimes_{R} c^{1} \otimes_{R} \cdots \otimes_{R} c^{n} \\
& +\sum_{i=1}^{n}(-1)^{i} c^{1} \otimes_{R} \cdots \otimes_{R} c^{i-1} \otimes_{R} \Delta_{\mathfrak{C}}\left(c^{i}\right) \otimes_{R} c^{i+1} \otimes_{R} \cdots \otimes_{R} c^{n} \\
& +(-1)^{n+1} c^{1} \otimes_{R} \cdots \otimes_{R} c^{n} \otimes_{R} g
\end{aligned}
$$

A direct computation shows that $d \circ d=0$ and that $d$ satisfies the Leibniz rule, hence $(\Omega(\mathfrak{C}), d)$ is a differential graded ring.

REMARK 3.1. In fact the above construction of a differential graded ring can be extended to a slightly more general situation. An element $g \in \mathfrak{C}$ is called a semi-grouplike element provided $\Delta_{\mathfrak{C}}(g)=g \otimes_{R} g$. Note that every coring has a semi-grouplike element (indeed, take $g=0$ ). Note also that the definition of a semi-grouplike element implies that $u=\epsilon_{\mathfrak{C}}(g) \in R$ is an idempotent in the centraliser of $g$ in $R$, i.e., $u^{2}=u$ and $u g=g u$. The above construction of a differential graded ring makes no use of the fact that $u=1_{R}$ for a grouplike element. Thus it holds even if the phrase " $g$ is a grouplike element" is 
replaced by " $g$ is a semi-grouplike element". The case of $g=0$ is of particular interest for the development of the Cartier cohomology of corings [18] as it corresponds to the cobar resolution of $\mathfrak{C}$.

Following Sweedler [26], given a ring $S$, a ring $R$ is called an $S$-ring or an algebra over $S$ if there is a ring map $S \rightarrow R$. Thus the notion of an $S$-ring extends the notion of an $S$-algebra to the case in which $S$ is neither commutative nor central in $R$. Extending further this notion to differential graded rings one says that $\Omega$ is an $S$-relative differential graded ring or a differential graded algebra over $S$ if there is a ring map $S \rightarrow R=\Omega^{0}$ such that $d$ is an $(S, S)$-bimodule map and $d(S)=0$.

Proposition 3.2. Let $\mathfrak{C}$ be an $R$-coring with a grouplike element $g$, and let $S=R_{g}^{\text {coc }}$. Then the differential graded ring $(\Omega(\mathfrak{C}), d)$ is an $S$-relative differential graded ring.

Proof. This proposition immediately follows from the facts that $S$ is a centraliser of $g$ in $R$ (hence $d(S)=0$ ) and that $\Delta_{\mathfrak{C}}$ is an $(S, S)$-bimodule map.

Example 3.3. Take a ring extension $S \rightarrow R$, the canonical Sweedler coring $\mathfrak{C}=$ $R \otimes_{S} R$ and a grouplike $g=1_{R} \otimes_{S} 1_{R}$. Then $\Omega^{n}(\mathfrak{C})=R \otimes_{S} R \otimes_{S} \ldots \otimes_{S} R(n+1$-times $)$, and $d^{n}=\sum_{i=0}^{n+1}(-1)^{i} e_{i}^{n}$, where

$$
e_{i}^{n}: r_{1} \otimes_{S} \ldots \otimes_{S} r_{n+1} \mapsto r_{1} \otimes_{S} \ldots \otimes_{S} r_{i} \otimes_{S} 1_{R} \otimes_{S} r_{i+1} \otimes_{S} \ldots \otimes_{S} r_{n+1},
$$

$i=0,1, \ldots, n+1$. This means that $\left(\Omega\left(R \otimes_{S} R\right), d\right)$ is the Amitsur complex associated to a ring extension $S \rightarrow R$ [2] (see also [3, Section 6]).

Motivated by Example 3.3 we call the cochain complex $(\Omega(\mathfrak{C}), d)$ the Amitsur complex associated to a coring $\mathfrak{C}$ and a grouplike $g$.

EXAMPLE 3.4. Let $\mathfrak{C}=A \otimes C$ be a coring associated to an entwining structure $(A, C)_{\psi}$ over $k$. Suppose that $A$ is an $(A, C)_{\psi}$-entwined module. Then $\Omega^{n}(\mathfrak{C})=A \otimes C^{\otimes n}$, and

$$
\begin{aligned}
d^{n}\left(a \otimes c_{1} \otimes \ldots \otimes c_{n}\right)= & a_{(0)} \otimes a_{(1)} \otimes c_{1} \otimes \ldots \otimes c_{n} \\
& +\sum_{i=1}^{n}(-1)^{i} a \otimes c_{1} \otimes \ldots \otimes \Delta\left(c_{i}\right) \otimes \ldots \otimes c_{n} \\
& +(-1)^{n+1} a \psi^{n}\left(c_{1} \otimes \ldots \otimes c_{n} \otimes 1_{(0)}\right) \otimes 1_{(1)}
\end{aligned}
$$

where $\psi^{n}=\left(\psi \otimes C^{\otimes n-1}\right) \circ \ldots \circ\left(C^{\otimes n-2} \otimes \psi \otimes C\right) \circ\left(C^{\otimes n-1} \otimes \psi\right)$. Note that the product in $\Omega(\mathfrak{C})$ reads

$$
\begin{aligned}
&\left(a \otimes c_{1} \otimes \ldots \otimes c_{m}\right)\left(a^{\prime} \otimes c_{m+1} \otimes \ldots \otimes c_{m+n}\right) \\
&=a \psi^{m}\left(c_{1} \otimes \ldots \otimes c_{m} \otimes a^{\prime}\right) \otimes c_{m+1} \otimes \ldots \otimes c_{m+n} .
\end{aligned}
$$

Proof. Only the last term in the expression for $d^{n}$ might require some explanation. We use the $\alpha$-notation $\psi(c \otimes a)=a_{\alpha} \otimes c^{\alpha}$ (summation over repeated indices understood) so that $\psi^{n}\left(c_{1} \otimes \ldots \otimes c_{n} \otimes a\right)=a_{\alpha_{n} \ldots \alpha_{1}} \otimes c_{1}^{\alpha_{1}} \otimes \ldots \otimes c_{n}^{\alpha_{n}}$. Then the last term in the expression for $d^{n}$ is obtained by the following chain of identifications:

$$
\begin{aligned}
\left(a \otimes c_{1} \otimes_{A} 1_{A} \otimes c_{2} \otimes_{A} \ldots \otimes_{A} 1_{A} \otimes c_{n}\right) \otimes_{A} 1_{(0)} \otimes 1_{(1)} \\
=a \otimes c_{1} \otimes_{A} 1_{A} \otimes c_{2} \otimes_{A} \ldots \otimes_{A} 1_{(0) \alpha_{n}} \otimes c_{n}^{\alpha_{n}} \otimes 1_{(1)}
\end{aligned}
$$




$$
\begin{aligned}
& =a \otimes c_{1} \otimes_{A} 1_{A} \otimes c_{2} \otimes_{A} \ldots \otimes_{A} 1_{(0)_{\alpha_{n} \alpha_{n-1}}} \otimes c_{n-1}^{\alpha_{n-1}} \otimes c_{n}^{\alpha_{n}} \otimes 1_{(1)} \\
& \ldots \\
& =a 1_{(0)_{\alpha_{n} \ldots \alpha_{1}}} \otimes c_{1}^{\alpha_{1}} \otimes \ldots \otimes c_{n}^{\alpha_{n}} \otimes 1_{(1)} .
\end{aligned}
$$

Similar chain leads to the product in $\Omega(\mathfrak{C})$.

Let $\mathfrak{C}$ be an $R$-coring with a grouplike element $g$, and let $S=R_{g}^{c o \mathfrak{C}}$. Recall from [6] that a pair $(\mathfrak{C}, g)$ is said to be a Galois coring if and only if there exists an $R$-coring isomorphism $\chi: R \otimes_{S} R \rightarrow \mathfrak{C}$ such that $\chi\left(1_{R} \otimes_{S} 1_{R}\right)=g$. The map $\chi$ is called a Galois isomorphism.

Proposition 3.5. The Amitsur complex of a Galois $R$-coring $(\mathfrak{C}, g)$ is acyclic provided $R$ is a faithfully flat left module of the subring $S$ of its $g$-coinvariants.

Proof. Since $R$ is a faithfully flat left $S$-module and the Amitsur complex $(\Omega(\mathfrak{C}), d)$ is a complex in the category of right $S$-modules, it suffices to show that the complex $\left(\Omega(\mathfrak{C}) \otimes_{S} R, d \otimes_{S} R\right)$ is acyclic. Let $\chi: R \otimes_{S} R \rightarrow \mathfrak{C}$ be the Galois isomorphism of corings and write $\chi^{-1}(c)=c^{(1)} \otimes_{S} c^{(2)}$ (summation understood), for all $c \in \mathfrak{C}$. First note that for all $c \in \mathfrak{C}, c^{(1)} g c^{(2)}=c$, and then compute

$$
\begin{aligned}
& \left(\mathfrak{C} \otimes_{R} \chi\right)\left(c^{(1)} g \otimes_{R} 1_{R} \otimes_{S} c^{(2)}-c_{(1)} \otimes_{R} c_{(2)}{ }^{(1)} \otimes_{S} c_{(2)}{ }^{(2)}\right) \\
& =c^{(1)} g \otimes_{R} g c^{(2)}-c_{(1)} \otimes_{R} c_{(2)}=\Delta_{\mathfrak{C}}\left(c^{(1)} g c^{(2)}-c\right)=0 .
\end{aligned}
$$

Since $\chi$ is bijective we conclude that for all $c \in \mathfrak{C}$,

$$
c^{(1)} g \otimes_{S} c^{(2)}=c_{(1)} \chi^{-1}\left(c_{(2)}\right)
$$

For any $n=1,2, \ldots$ consider an additive map $h^{n}: \Omega^{n}(\mathfrak{C}) \otimes_{S} R \rightarrow \Omega^{n-1}(\mathfrak{C}) \otimes_{S} R$, $h^{n}: c^{1} \otimes_{R} \ldots \otimes_{R} c^{n} \otimes_{S} r \mapsto(-1)^{n} c^{1} \otimes_{R} \ldots \otimes_{R} c^{n-1} \chi^{-1}\left(c^{n} r\right)$. We will show that the collection $h$ of all such $h^{n}$ is a contracting homotopy for $d \otimes_{S} R$. On the one hand we have

$$
\begin{aligned}
h^{n+1} & \left(d^{n}\left(c^{1} \otimes_{R} \ldots \otimes_{R} c^{n}\right) \otimes_{S} r\right)=(-1)^{n+1} g \otimes_{R} c^{1} \otimes_{R} \ldots \otimes_{R} c^{n-1} \chi^{-1}\left(c^{n} r\right) \\
& +\sum_{i=1}^{n-1}(-1)^{n+i+1} c^{1} \otimes_{R} \ldots \otimes_{R} \Delta_{\mathfrak{C}}\left(c^{i}\right) \otimes_{R} \ldots \otimes_{R} c^{n-1} \chi^{-1}\left(c^{n} r\right) \\
& -c^{1} \otimes_{R} \cdots \otimes_{R} c^{n}(1) \chi^{-1}\left(c^{n}{ }_{(2)} r\right)+c^{1} \otimes_{R} \cdots \otimes_{R} c^{n} \chi^{-1}(g r) \\
= & (-1)^{n+1} g \otimes_{R} c^{1} \otimes_{R} \ldots \otimes_{R} c^{n-1} \chi^{-1}\left(c^{n} r\right) \\
& +\sum_{i=1}^{n-1}(-1)^{n+i+1} c^{1} \otimes_{R} \ldots \otimes_{R} \Delta_{\mathfrak{C}}\left(c^{i}\right) \otimes_{R} \cdots \otimes_{R} c^{n-1} \chi^{-1}\left(c^{n} r\right) \\
& -c^{1} \otimes_{R} \cdots \otimes_{R} c^{n(1)} g \otimes_{S} c^{n(2)} r+c^{1} \otimes_{R} \cdots \otimes_{R} c^{n} \otimes_{S} r
\end{aligned}
$$

where we have used equation $(*)$ and the fact that $\chi^{-1}(g)=1_{R} \otimes_{S} 1_{R}$. On the other hand,

$$
\begin{aligned}
& d^{n-1}\left(h^{n}\left(c^{1} \otimes_{R} \ldots \otimes_{R} c^{n}\right) \otimes_{S} r\right)=(-1)^{n} g \otimes_{R} c^{1} \otimes_{R} \ldots \otimes_{R} c^{n-1} \chi^{-1}\left(c^{n} r\right) \\
& \quad+\sum_{i=1}^{n-1}(-1)^{n+i} c^{1} \otimes_{R} \ldots \otimes_{R} \Delta_{\mathfrak{C}}\left(c^{i}\right) \otimes_{R} \ldots \otimes_{R} c^{n-1} \chi^{-1}\left(c^{n} r\right) \\
& +c^{1} \otimes_{R} \cdots \otimes_{R} c^{n(1)} g \otimes_{S} c^{n(2)} r .
\end{aligned}
$$


Thus $h^{n+1} d^{n}+d^{n-1} h^{n}=\Omega^{n}(\mathfrak{C}) \otimes_{S} R$. This means that $d \otimes_{S} R$ is homotopic to the identity, so the complex $\left(\Omega(\mathfrak{C}) \otimes_{S} R, d \otimes_{S} R\right)$ is acyclic, and therefore the Amitsur complex is acyclic by virtue of the fact that the functor $-\otimes_{S} R$ reflects exact sequences (for $R$ is a faithfully flat left $S$-module).

In fact, since there is a ring map $S \rightarrow R$, hence a map $S \rightarrow \Omega(\mathfrak{C})$, if $S \rightarrow R$ is faithfully flat and $(\mathfrak{C}, g)$ is a Galois coring then the associated Amitsur complex $\Omega(\mathfrak{C})$ is a resolution of $S$. Therefore Proposition 3.5 can be seen as a coring version of a well-known fact in the faithfully flat descent theory (cf. [3, Proposition 6.2]).

4. Connections and comodules. The category of comodules of a coring with a grouplike element can be naturally described in terms of connections over a ring. Let $S \rightarrow R$ be a ring extension, and let $\Omega$ be an $S$-relative differential graded ring with $R=\Omega^{0}$. Recall from [12] that a connection in a right $R$-module $M$ is a right $S$-linear map $\nabla: M \otimes_{R} \Omega^{\bullet} \rightarrow M \otimes_{R} \Omega^{\bullet+1}$ which satisfies the Leibniz rule, i.e., such that for all $\omega \in M \otimes_{R} \Omega^{k}$ and $\omega^{\prime} \in \Omega, \nabla\left(\omega \omega^{\prime}\right)=\nabla(\omega) \omega^{\prime}+(-1)^{k} \omega d\left(\omega^{\prime}\right)$. A curvature of a connection $\nabla$ is a right $S$-linear map $F_{\nabla}: M \rightarrow M \otimes_{R} \Omega^{2}$, defined as the restriction of $\nabla \circ \nabla$ to $M$, i.e., $F_{\nabla}=\left.\nabla \circ \nabla\right|_{M}$. A connection is said to be flat if its curvature is identically equal to 0 .

It is important to note that a connection is fully determined by its restriction to the module $M$. Indeed, any element of $M \otimes_{R} \Omega$ is a sum of terms of the form $m \otimes_{R} \omega$ with $m \in M$ and $\omega \in \Omega$. Now, viewing $m$ as an element of $M \otimes_{R} \Omega^{0}$, and using the Leibniz rule, the action of $\nabla$ on $m \otimes_{R} \omega$ reads $\nabla\left(m \otimes_{R} \omega\right)=\nabla(m) \omega+m \otimes_{R} d(\omega)$.

To describe a relationship between connections and comodules of a coring we first need to introduce an appropriate differential graded ring [25, Lemma 1].

Proposition 4.1. Let $\mathfrak{C}$ be an $R$-coring with a grouplike $g \in \mathfrak{C}$ and let $S$ be the subring of $g$-coinvariants. Then the associated Amitsur complex $(\Omega(\mathfrak{C}), d)$ restricts to the $S$-relative differential graded ring $(\Omega(\mathfrak{C} / S), d)$ with $\Omega^{0}(\mathfrak{C} / S)=R$ and

$$
\Omega^{n}(\mathfrak{C} / S)=\operatorname{ker} \epsilon_{\mathfrak{C}} \otimes_{R} \operatorname{ker} \epsilon_{\mathfrak{C}} \otimes_{R} \ldots \otimes_{R} \operatorname{ker} \epsilon_{\mathfrak{C}},
$$

(ker $\epsilon_{\mathfrak{C}}$ taken $n$-times).

Proof. The key observation here is that, first, for all $r \in R, \epsilon_{\mathfrak{C}}(d(r))=\epsilon_{\mathfrak{C}}(g r)-$ $\epsilon_{\mathfrak{C}}(r g)=r-r=0$, and, second, for any $c^{1}, \ldots, c^{n} \in \operatorname{ker} \epsilon_{\mathfrak{C}}$, the Amitsur coboundary operator $d^{n}$ can be written equivalently as

$$
\begin{aligned}
d^{n}\left(c^{1} \otimes_{R} \ldots \otimes_{R} c^{n}\right)= & \sum_{i=1}^{n}(-1)^{i} c^{1} \otimes_{R} \ldots \otimes_{R} c^{i-1} \\
& \otimes_{R}\left(c^{i}(1)-g \epsilon_{\mathfrak{C}}\left(c^{i}(1)\right)\right) \otimes_{R}\left(c^{i}{ }_{(2)}-\epsilon_{\mathfrak{C}}\left(c^{i}(2)\right) g\right) \\
& \otimes_{R} c^{i+1} \otimes_{R} \ldots \otimes_{R} c^{n} .
\end{aligned}
$$

This expression shows immediately that the image of $d^{n}$ applied to $\left(\operatorname{ker} \epsilon_{\mathfrak{C}}\right)^{\otimes_{R} n}$ is in $\left(\operatorname{ker} \epsilon_{\mathfrak{C}}\right)^{\otimes_{R} n+1}$ as required.

Thus we have constructed an $S$-relative differential graded ring $\Omega(\mathfrak{C} / S)$, which is termed a ring of $\mathfrak{C}$-valued differential forms on $R$. This construction has an interesting 
converse. Let $\Omega$ be a differential graded ring with $\Omega^{0}=R$, and $\Omega^{n}=\Omega^{1} \otimes_{R} \Omega^{1} \otimes_{R} \ldots \otimes_{R} \Omega^{1}$ (n-times). Consider a left $R$-module $\mathfrak{C}=R g \oplus \Omega^{1}$. One easily checks that $\mathfrak{C}$ can be made into an $(R, R)$-bimodule with a right multiplication $(r g+\omega) r^{\prime}=r r^{\prime} g+r d\left(r^{\prime}\right)+\omega r^{\prime}$ for all $r, r^{\prime} \in R, \omega \in \Omega^{1}$. Then $\mathfrak{C}$ is an $R$-coring with the coproduct and counit

$$
\Delta_{\mathfrak{C}}(r g)=r g \otimes_{R} g, \quad \Delta_{\mathfrak{C}}(\omega)=g \otimes_{R} \omega+\omega \otimes_{R} g-d(\omega), \quad \epsilon_{\mathfrak{C}}(r g+\omega)=r,
$$

for all $r \in R$ and $\omega \in \Omega^{1}$. The coassociativity of $\Delta_{\mathfrak{C}}$ follows from the equality $d\left(\omega^{(1)}\right) \otimes_{R}$ $\omega^{(2)}+\omega^{(1)} \otimes_{R} d\left(\omega^{(2)}\right)=0$, where $d(\omega)=\omega^{(1)} \otimes_{R} \omega^{(2)}$ is a notation. This is a consequence of the Leibniz rule and the nilpotency of $d$. Clearly, $g$ is a grouplike element and $\Omega=\Omega(\mathfrak{C} / S)$, where $S=\operatorname{ker}\left(d: R \rightarrow \Omega^{1}\right)$. Note also that $d(r)=g r-r g$. In particular some differential calculi of noncommutative geometry based on Dirac operators or Fredholm modules, lead to corings with grouplike elements.

Given a ring extension $S \rightarrow R$ there is an associated universal differential graded algebra over $S$ generated by the $S$-ring $R$ known as an algebra of $S$-relative differential forms $\Omega_{S} R$ [13]. Let $R / S$ denote the cokernel of the ring homomorphism $S \rightarrow R$ viewed as a map of $(S, S)$-bimodules, and let $\pi: R \rightarrow R / S$ be the canonical map of $(S, S)$ bimodules. Thus $R / S$ is an $S$-bimodule and for any $n \in \mathbb{N} \cup\{0\}$ one can consider an $(R, S)$-bimodule

$$
\Omega_{S}^{n} R=R \otimes_{S}(R / S)^{\otimes_{S} n}=R \otimes_{S} R / S \otimes_{S} R / S \otimes_{S} \cdots \otimes_{S} R / S,
$$

and combine them into a direct sum $\Omega_{S} R=\oplus_{n=0}^{\infty} \Omega_{S}^{n} R . \Omega_{S} R$ is a cochain complex with a coboundary operator

$$
d: \Omega_{S}^{\bullet} R \rightarrow \Omega_{S}^{\bullet+1} R, \quad r_{0} \otimes_{S} r_{1} \otimes_{S} \ldots \otimes_{S} r_{n} \mapsto 1_{R} \otimes_{S} \pi\left(r_{0}\right) \otimes_{S} r_{1} \otimes_{S} \ldots \otimes_{S} r_{n} .
$$

It is clear that $d \circ d=0$ since $S \rightarrow R$ as a ring homomorphism is a unit preserving map so that $\pi\left(1_{R}\right)=0$. Less trivial is the observation that $\left(\Omega_{S} R, d\right)$ is an $S$-relative differential graded ring. The product in $\Omega_{S} R$ is given by the formula

$$
\left(r_{0}, \ldots, r_{n}\right)\left(r_{n+1}, \ldots, r_{m}\right)=\sum_{i=0}^{n}(-1)^{n-i}\left(r_{0}, \ldots, r_{i-1}, r_{i} \cdot r_{i+1}, r_{i+2}, \ldots, r_{m}\right)
$$

where we write $\left(r_{0}, \ldots, r_{n}\right)$ for $r_{0} \otimes_{S} \ldots \otimes_{S} r_{n}$ etc., to relieve the notation. Also, the notation $r_{i} \cdot r_{i+1}$ is a formal expression that is to be understood as follows. Take any $r_{i}^{\prime} \in \pi^{-1}\left(r_{i}\right)$ and $r_{i+1}^{\prime} \in \pi^{-1}\left(r_{i+1}\right), i=1, \ldots n-1$. Then

$$
r_{i} \cdot r_{i+1}= \begin{cases}r_{i} r_{i+1}^{\prime} & \text { for } i=0 \\ \pi\left(r_{i}^{\prime} r_{i+1}^{\prime}\right) & \text { for } 0<i<n \\ \pi\left(r_{i}^{\prime} r_{i+1}\right) & \text { for } i=n\end{cases}
$$

Although $r_{i} \cdot r_{i+1}$ depends on the choice of the $r_{i}^{\prime}$, it can be easily shown that the product of cochains does not. It can also be shown that the above expression defines an associative product and that $d$ satisfies the graded Leibniz rule. The details can be found in [13]. The algebra of $S$-relative differential forms has the following universality property. Given any graded differential ring $\Omega=\oplus_{n} \Omega^{n}$ and a ring homomorphism $u: R \rightarrow \Omega^{0}$ such that $d(u(s))=0$ for all $s \in S$, there exists a unique differential graded ring homomorphism $u_{*}: \Omega_{S} R \rightarrow \Omega$ extending $u$. This means, in particular, that the identity map $R \rightarrow R$ 
extends to a map of $S$-relative differential graded rings $\Omega_{S} R \rightarrow \Omega(\mathfrak{C} / S)$. This can be understood purely in terms of coring valued differential forms since we have the following

Proposition 4.2. Let $S \rightarrow R$ be a ring extension. Take the canonical Sweedler coring $\mathfrak{C}=R \otimes_{S} R$, and a grouplike element $g=1_{R} \otimes_{S} 1_{R}$. Then the differential graded algebra over $S$ of $\mathfrak{C}$-valued differential forms is isomorphic to the algebra of $S$-relative differential forms $\Omega_{S} R$.

Proof. We first explicitly describe the structure of $\mathfrak{C}=R \otimes_{S} R$-valued differential forms. Note that since the counit of the canonical coring coincides with the product map $\mu_{R / S}: R \otimes_{S} R \rightarrow R, r \otimes_{S} r^{\prime} \mapsto r r^{\prime}$, we have $\Omega^{1}(\mathfrak{C} / S)=\operatorname{ker} \epsilon_{\mathfrak{C}}=\operatorname{ker} \mu_{R / S}$. Clearly, the Amitsur 0-differential $d: r \mapsto 1_{R} \otimes_{S} r-r \otimes_{S} 1_{R}$, has values restricted to ker $\mu_{R / S}$. Note that $\Omega^{n}(\mathfrak{C} / S)=\left(\operatorname{ker} \mu_{R / S}\right)^{\otimes_{R} n}$. Thus if we could show that $\operatorname{ker} \mu_{R / S} \cong R \otimes_{S} R / S$ as $(R, R)$-bimodules, then we would have the required form of $\Omega^{n}(\mathfrak{C} / S)$. Indeed, the iteration

$$
\begin{aligned}
\Omega^{n}(\mathfrak{C} / S) & =\Omega^{n-1}(\mathfrak{C} / S) \otimes_{R} \operatorname{ker} \mu_{R / S} \\
& \cong \Omega^{n-1}(\mathfrak{C} / S) \otimes_{R} R \otimes_{S} R / S \cong \Omega^{n-1}(\mathfrak{C} / S) \otimes_{S} R / S
\end{aligned}
$$

repeated $n$-times would yield the desired result. Note that $\operatorname{ker} \mu_{R / S} \cong R \otimes_{S} R / S$ as $(R, S)$-bimodules via the map $\theta: \operatorname{ker} \mu_{R / S} \rightarrow R \otimes_{S} R / S, \sum_{i} r_{i} \otimes_{S} r_{i}^{\prime} \mapsto \sum_{i} r_{i} \otimes_{S} \pi\left(r_{i}^{\prime}\right)$ with the inverse $\theta^{-1}: r \otimes_{S} \pi\left(r^{\prime}\right)=r \otimes_{S} r^{\prime}-r r^{\prime} \otimes_{S} 1_{R}$. Note also that the map $\theta^{-1}$ does not depend on the choice of $r^{\prime}$ in the inverse image of $\pi\left(r^{\prime}\right)$. Indeed, if $\pi\left(r^{\prime}\right)=0$ then $r^{\prime}=s 1_{R}$ with $s \in S$ and hence $r \otimes_{S} r^{\prime}-r r^{\prime} \otimes_{S} 1_{R}=r \otimes_{S} s 1_{R}-r s \otimes_{S} 1_{R}=0$ as needed. The right $R$-module structure of $R \otimes_{S} R / S$ is derived from the product in $\Omega_{S} R$, i.e., $\left(r_{0} \otimes_{S} \pi\left(r_{1}\right)\right) r=-r_{0} r \otimes_{S} \pi\left(r_{1}\right)+r_{0} \otimes_{S} \pi\left(r_{1} r\right)$ and is well defined (does not depend on the choice of $r_{1}$ ) by the similar argument as above. Clearly $\theta$ and $\theta^{-1}$ are maps of right $R$-modules. The isomorphism $\theta$ extends to cochains of all degrees and one can easily check that it provides an isomorphism of $S$-relative graded differential algebras. This isomorphism involves projections $\pi$ in all bar the first tensorand and thus maps all the Amitsur operators $e_{i}^{n}$ with $i>0$ in Example 3.3 to 0. Thus the resulting differential $d$ has the form $d: r_{0} \otimes_{S} r_{1} \otimes_{S} \ldots \otimes_{S} r_{n} \mapsto 1_{R} \otimes_{S} \pi\left(r_{0}\right) \otimes_{S} r_{1} \otimes_{S} \ldots \otimes_{S} r_{n}$, as required, and the proof of the proposition is completed.

Next we address the problem of existence of $\Omega(\mathfrak{C} / S)$-valued connections.

TheOREM 4.3. Let $\mathfrak{C}$ be an $R$-coring with a grouplike element $g \in \mathfrak{C}$, let $S$ be the subring of $g$-coinvariants of $R$, and let $\Omega(\mathfrak{C} / S)$ be the ring of $\mathfrak{C}$-valued differential forms on $R$. A right $R$-module admits an $\Omega(\mathfrak{C} / S)$ valued connection if and only if $M \otimes_{R} \epsilon_{\mathfrak{C}}$ is a right $R$-module retraction.

Proof. Given a connection $\nabla: M \rightarrow M \otimes_{R} \Omega^{1}(\mathfrak{C} / S)$ define an additive map

$$
j_{\nabla}: M \rightarrow M \otimes_{R} \mathfrak{C}, \quad m \mapsto \nabla(m)+m \otimes_{R} g .
$$

Since $\operatorname{Im}(\nabla) \subseteq M \otimes_{R}$ ker $\epsilon_{\mathfrak{C}}$ and $\epsilon_{\mathfrak{C}}(g)=1_{R}$, the map $j_{\nabla}$ is an additive section of $M \otimes_{R} \epsilon_{\mathfrak{C}}$. Furthermore for all $m \in M$ and $r \in R$,

$$
\begin{aligned}
j_{\nabla}(m r) & =\nabla(m r)+m r \otimes_{R} g=\nabla(m) r+m \otimes_{R} d(r)+m r \otimes_{R} g \\
& =\nabla(m) r+m \otimes_{R} g r-m \otimes_{R} r g+m \otimes_{R} r g=j_{\nabla}(m) r .
\end{aligned}
$$


where we used the Leibniz rule for $\nabla$ to obtain the second equality. Thus $j_{\nabla}$ is a right $R$-linear section of $M \otimes_{R} \epsilon_{\mathfrak{C}}$.

Conversely, suppose $j: M \rightarrow M \otimes_{R} \mathfrak{C}$ is a right $R$-linear section of $M \otimes_{R} \epsilon_{\mathfrak{C}}$, and define an additive map

$$
\nabla_{j}: M \rightarrow M \otimes_{R} \Omega^{1}(\mathfrak{C} / S)=M \otimes_{R} \operatorname{ker} \epsilon_{\mathfrak{C}}, \quad m \mapsto j(m)-m \otimes_{R} g .
$$

Note that $\nabla_{j}$ is well-defined since the fact that $j$ is a section implies that for all $m \in M$, $\sum_{i} m^{i} \epsilon_{\mathfrak{C}}\left(c^{i}\right)=m$, where $\sum_{i} m^{i} \otimes_{R} c^{i}=j(m)$. Therefore

$$
\nabla_{j}(m)=\sum_{i}\left(m^{i} \otimes_{R} c^{i}-m^{i} \epsilon_{\mathfrak{C}}\left(c^{i}\right) \otimes_{R} g\right)=\sum_{i} m^{i} \otimes_{R}\left(c^{i}-\epsilon_{\mathfrak{C}}\left(c^{i}\right) g\right),
$$

and each $c^{i}-\epsilon_{\mathfrak{C}}\left(c^{i}\right) g \in \operatorname{ker} \epsilon_{\mathfrak{C}}$. Finally for all $m \in M$ and $r \in R$ we have

$$
\begin{aligned}
\nabla_{j}(m r) & =j(m r)-m r \otimes_{R} g=j(m) r-m \otimes_{R} r g \\
& =j(m) r-m \otimes_{R} g r+m \otimes_{R} g r-m \otimes_{R} r g=\nabla_{j}(m) r+m \otimes_{R} d r,
\end{aligned}
$$

so that $\nabla_{j}$ satisfies the Leibniz rule for a connection as required.

In particular Theorem 4.3 implies that if a right $R$-module $M$ admits an $\Omega(\mathfrak{C} / S)$ valued connection then $M$ is a direct summand of a right $R$-module $M \otimes_{R} \mathfrak{C}$. As a special case of Theorem 4.3 one can take an algebra $A$ over a field $k$, view it as an extension of $k$, and thus consider the corresponding Sweedler $A$-coring $\mathfrak{C}=A \otimes A$ and a grouplike $g=1_{A} \otimes 1_{A}$. In this case the $g$-coinvariants of $A$ are simply equal to $k$, and $\Omega(\mathfrak{C} / k)=\Omega A$ is a universal differential envelope of $A$ by Proposition 4.2. By the canonical identification $M \otimes_{A} A \otimes A \cong M \otimes A$, for any $M \in \mathbf{M}_{A}$, the map $M \otimes_{A} \epsilon_{\mathfrak{C}}$ coincides with the action of $A$ on $M, m \otimes a \mapsto m a$. The action is an $A$-module retraction if and only if $M$ is a projective right $A$-module. Thus, as a special case of Theorem 4.3, we obtain the classic result of Cuntz and Quillen [13, Corollary 8.2] which relates the existence of (universal) connections to projectivity.

The importance of coring valued differential forms in relation to comodules of a coring is revealed by the following theorem.

TheOREM 4.4. Let $\mathfrak{C}$ be an $R$-coring with a grouplike $g \in \mathfrak{C}$, let $S$ be the subring of $g$-coinvariants, and let $\Omega(\mathfrak{C} / S)$ be the ring of $\mathfrak{C}$-valued differential forms on $R$. Then a right $R$-module $\mathfrak{M}$ is a right $\mathfrak{C}$-comodule if and only if it admits a flat connection $\nabla: \mathfrak{M} \rightarrow \mathfrak{M} \otimes_{R} \Omega(\mathfrak{C} / S)$

Proof. Suppose $\mathfrak{M}$ is a right $\mathfrak{C}$-comodule with a coaction $\rho^{\mathfrak{M}}$ and define

$$
\nabla: \mathfrak{M} \rightarrow \mathfrak{M} \otimes_{R} \Omega^{1}(\mathfrak{C} / S), \quad m \mapsto \rho^{\mathfrak{M}}(m)-m \otimes_{R} g .
$$

Since $\rho^{\mathfrak{M}}$ is a right $R$-module splitting of $\mathfrak{M} \otimes_{R} \epsilon_{\mathfrak{C}}$, the map $\nabla$ is a connection by Theorem 4.3.

We can now compute the curvature $F_{\nabla}$ of $\nabla$. Take any $m \in \mathfrak{M}$ and compute

$$
\begin{aligned}
F_{\nabla}(m) & =\nabla\left(m_{(0)} \otimes_{R} m_{(1)}-m \otimes_{R} g\right) \\
& =\nabla\left(m_{(0)}\right) \otimes_{R} m_{(1)}+m_{(0)} \otimes_{R} d m_{(1)}-\nabla(m) \otimes_{R} g+m \otimes_{R} d g=0,
\end{aligned}
$$

by the coassociativity of $\rho^{\mathfrak{M}}$ and the definition of $d$. 
Conversely, suppose $\mathfrak{M}$ is a right $R$-module with a flat connection $\nabla: \mathfrak{M} \rightarrow \mathfrak{M} \otimes_{R}$ ker $\epsilon_{\mathfrak{C}}$. For any $m \in \mathfrak{M}$, write $\nabla(m)=\sum_{i} m^{i} \otimes_{R} c^{i}$. Then the flatness of $\nabla$ means that $0=\nabla\left(\sum_{i} m^{i} \otimes_{R} c^{i}\right)=\sum_{i} \nabla\left(m^{i}\right) \otimes_{R} c^{i}+\sum_{i} m^{i} \otimes_{R} d\left(c^{i}\right)$, i.e.,

$\sum_{i} m^{i} \otimes_{R} c^{i}{ }_{(1)} \otimes_{R} c^{i}{ }_{(2)}=\sum_{i j} m^{i j} \otimes_{R} \tilde{c}^{j} \otimes_{R} c^{i}+\sum_{i} m^{i} \otimes_{R} g \otimes_{R} c^{i}+\sum_{i} m^{i} \otimes_{R} c^{i} \otimes_{R} g$,

where $\nabla\left(m^{i}\right)=\sum_{j} m^{i j} \otimes_{R} \tilde{c}^{j}$.

Now define an additive map $\rho^{\mathfrak{M}}: \mathfrak{M} \rightarrow \mathfrak{M} \otimes_{R} \mathfrak{C}, m \mapsto \nabla(m)+m \otimes_{R} g$. Note that $\rho^{\mathfrak{M}}$ coincides with the map $j_{\nabla}$ constructed in the proof of Theorem 4.3, and thus it is a right $R$-module map such that $\left(\mathfrak{M} \otimes_{R} \epsilon_{\mathfrak{C}}\right) \rho^{\mathfrak{M}}(m)=m$. Therefore it only remains to be shown that $\rho^{\mathfrak{M}}$ is coassociative. Since explicitly $\rho^{\mathfrak{M}}(m)=\sum_{i} m^{i} \otimes_{R} c^{i}+m \otimes_{R} g$, we have

$$
\begin{aligned}
\left(\rho^{\mathfrak{M}} \otimes_{R} \mathfrak{C}\right) \rho^{\mathfrak{M}}(m)= & \sum_{i} \rho^{\mathfrak{M}}\left(m^{i}\right) \otimes_{R} c^{i}+\rho^{\mathfrak{M}}(m) \otimes_{R} g \\
= & \sum_{i j} m^{i j} \otimes_{R} \tilde{c}^{j} \otimes_{R} c^{i}+\sum_{i} m^{i} \otimes_{R} g \otimes_{R} c^{i} \\
& +\sum_{i} m^{i} \otimes_{R} c^{i} \otimes_{R} g+m \otimes_{R} g \otimes_{R} g \\
= & \sum_{i} m^{i} \otimes_{R} c^{i}{ }_{(1)} \otimes_{R} c^{i}{ }_{(2)}+m \otimes_{R} g \otimes_{R} g=\left(\mathfrak{M} \otimes_{R} \Delta_{\mathfrak{C}}\right) \rho^{\mathfrak{M}}(m)
\end{aligned}
$$

where we used the flatness of $\nabla$ to derive the penultimate equality. This proves that $\rho^{\mathfrak{M}}$ is a coassociative coaction, and hence $\mathfrak{M}$ is a right $\mathfrak{C}$-comodule.

In the proof of Theorem 4.4 we have constructed two assignments. Given a right $R$-module $\mathfrak{M}$, to every right $\mathfrak{C}$-coaction $\rho^{\mathfrak{M}}$ one assigns a flat connection $\nabla_{\rho^{\mathfrak{M}}}$ defined by $\nabla_{\rho^{\mathfrak{M}}}: m \mapsto \rho^{\mathfrak{M}}(m)-m \otimes_{R} g$. Conversely to any flat connection $\nabla$ one assigns a right $\mathfrak{C}$-coaction $\rho_{\nabla}^{\mathfrak{M}}: m \mapsto \nabla(m)+m \otimes_{R} g$. Clearly these assignments are inverses of each other and hence establish an isomorphism of sets of flat connections and right $\mathfrak{C}$-comodule structures. In fact Theorem 4.4 describes an isomorphism of categories.

Consider a ring extension $S \rightarrow R$ and an $S$-relative differential graded ring $\Omega$ with $\Omega^{0}=R$. A category of (right) connections with values in $\Omega$, denoted by $\operatorname{Conn}(R / S, \Omega$ ) consists of pairs $(M, \nabla)$ where $M$ is a right $R$-module and $\nabla: M \otimes_{R} \Omega^{\bullet} \rightarrow M \otimes_{R} \Omega^{\bullet+1}$ is a connection. A morphism $(M, \nabla) \rightarrow\left(N, \nabla^{\prime}\right)$ in $\operatorname{Conn}(R / S, \Omega)$ is a right $R$-module map $f: M \rightarrow N$ making the diagram

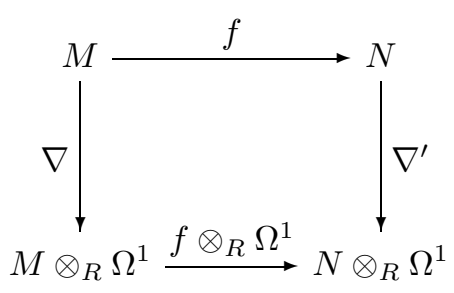

commute. Note that the Leibniz rule renders also the following diagram commutative: 


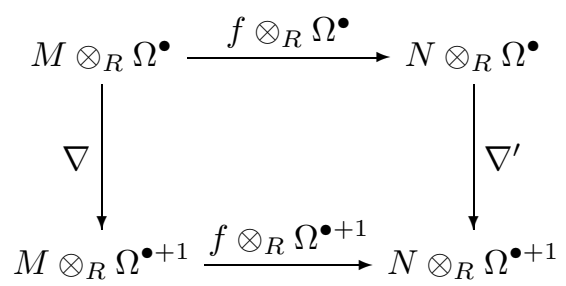

In particular we can consider the diagram

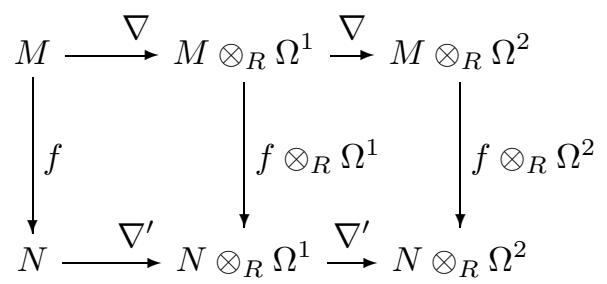

in which both left and right squares commute. This implies that the outer rectangle is commutative, hence $F^{\prime} \circ f=\left(f \otimes_{R} \Omega^{2}\right) \circ F$, where $F$ is the curvature of $\nabla$ and $F^{\prime}$ is the curvature of $\nabla^{\prime}$. This shows that $\operatorname{Conn}(R / S, \Omega)$ contains a full subcategory $\operatorname{Conn}_{0}(R / S, \Omega)$ of flat connections. Objects of $\mathbf{C o n n}_{0}(R / S, \Omega)$ are pairs $(M, \nabla)$ where $M$ is a right $R$-module and $\nabla$ is a flat connection.

Now Theorem 4.4 leads to the following

TheOREM 4.5. Let $\mathfrak{C}$ be an $R$-coring with a grouplike element $g \in \mathfrak{C}$, let $S$ be the subring of $g$-coinvariants, and let $\Omega(\mathfrak{C} / S)$ be the ring of $\mathfrak{C}$-valued differential forms on $R$. Then $\mathbf{M}_{R}^{\mathfrak{C}}$ is isomorphic to $\mathbf{C o n n}_{0}(R / S, \Omega(\mathfrak{C} / S))$.

Proof. On objects, the isomorphism is provided by the assignment constructed in Theorem 4.4, while on morphisms $\mathfrak{f}: \mathfrak{M} \rightarrow \mathfrak{N}, \mathfrak{f} \leftrightarrow \mathfrak{f}$. Indeed, if $\mathfrak{f}$ is a morphism of right $\mathfrak{C}$-comodules then for all $m \in \mathfrak{M}$,

$$
\begin{aligned}
\nabla_{\rho^{\mathfrak{N}}} \circ \mathfrak{f}(m) & =\rho^{\mathfrak{N}} \circ \mathfrak{f}(m)-\mathfrak{f}(m) \otimes_{R} g \\
& =\left(\mathfrak{f} \otimes_{R} \mathfrak{C}\right)\left(\rho^{\mathfrak{M}}(m)-m \otimes_{R} g\right)=\left(\mathfrak{f} \otimes_{R} \mathfrak{C}\right) \circ \nabla_{\rho^{\mathfrak{M}}}(m) .
\end{aligned}
$$

Conversely, if $\mathfrak{f}$ is a morphism $(\mathfrak{M}, \nabla) \rightarrow\left(\mathfrak{N}, \nabla^{\prime}\right)$ in $\operatorname{Conn}_{0}(R / S, \Omega(\mathfrak{C} / S))$ then

$$
\begin{aligned}
\rho_{\nabla^{\prime}}^{\mathfrak{N}} \circ \mathfrak{f}(m) & =\nabla^{\prime}(\mathfrak{f}(m))+\mathfrak{f}(m) \otimes_{R} g=\left(\mathfrak{f} \otimes_{R} \mathfrak{C}\right) \circ \nabla(m)+\mathfrak{f}(m) \otimes_{R} g \\
& =\left(\mathfrak{f} \otimes_{R} \mathfrak{C}\right) \circ\left(\nabla(m)+m \otimes_{R} g\right)=\left(\mathfrak{f} \otimes_{R} \mathfrak{C}\right) \circ \rho_{\nabla}^{\mathfrak{M}} .
\end{aligned}
$$

This completes the proof of the theorem.

EXAmple 4.6. The ring $R$ admits a flat connection, $\nabla(r)=g r-r g=d(r)$.

ExAmple 4.7. Let $(A, C)_{\psi}$ be an entwining structure over $k$, and let $\mathfrak{C}=A \otimes C$ be the associated $A$-coring. Suppose $A$ is an $(A, C)_{\psi}$-entwined module. Then $A \otimes C^{\otimes n}$, $n=1,2, \ldots$ has a flat connection

$$
\nabla\left(a \otimes c_{1} \otimes \ldots \otimes c_{n}\right)=a \otimes c_{1} \otimes \ldots \otimes \Delta\left(c_{n}\right)-a \psi^{n}\left(c_{1} \otimes \ldots \otimes c_{n} \otimes 1_{(0)}\right) \otimes 1_{(1)},
$$

where $\psi^{n}: C^{\otimes n} \otimes A \rightarrow A \otimes C^{\otimes n}$ is defined in Example 3.4.

Proof. This follows immediately from the fact that $A \otimes C^{\otimes n}$ is a right $\mathfrak{C}$-comodule (or, equivalently, an $(A, C)_{\psi}$-entwined module) [4]. 
Dually we have

ExAmple 4.8. Let $(A, C)_{\psi}$ be an entwining structure over $k$, let $\mathfrak{C}=A \otimes C$ be the associated $A$-coring. Suppose $A$ is an $(A, C)_{\psi}$-entwined module. Then $C \otimes A^{\otimes n}$, $n=1,2, \ldots$ has a flat connection

$$
\nabla\left(c \otimes a^{1} \otimes \ldots \otimes a^{n}\right)=c_{(1)} \otimes \psi_{n}\left(c_{(2)} \otimes a^{1} \otimes \ldots \otimes a^{n}\right)-c \otimes a^{1} \otimes \ldots \otimes a^{n} 1_{(0)} \otimes 1_{(1)},
$$
where $\psi_{n}=\left(A^{\otimes n-1} \otimes \psi\right) \circ \ldots \circ\left(A \otimes \psi \otimes A^{\otimes n-2}\right) \circ\left(\psi \otimes A^{\otimes n-1}\right): C \otimes A^{\otimes n} \rightarrow A^{\otimes n} \otimes C$.

Acknowledgements. I would like to thank EPSRC for an Advanced Research Fellowship.

\section{References}

[1] J. Y. Abuhlail, Rational modules for corings, preprint, 2001.

[2] S. Amitsur, Simple algebras and cohomology groups of arbitrary fields, Trans. Amer. Math. Soc. 90 (1959), 73-112.

[3] M. Artin, On Azumaya algebras and finite representations of rings, J. Algebra 11 (1969), $532-563$.

[4] T. Brzeziński, On modules associated to coalgebra-Galois extensions, J. Algebra 215 (1999), 290-317.

[5] T. Brzeziński, The cohomology structure of an algebra entwined with a coalgebra, J. Algebra 235 (2001), 176-202.

[6] T. Brzeziński, The structure of corings. Induction functors, Maschke-type theorem, and Frobenius and Galois-type properties, Algebras Representation Theory 5 (2002), 389-410.

[7] T. Brzeziński, Towers of corings, Comm. Algebra, in press. Preprint arXiv: math.RA/ 0201014, (2002).

[8] T. Brzeziński and S. Majid, Coalgebra bundles, Comm. Math. Phys. 191 (1998), 467-492.

[9] S. Caenepeel, G. Militaru and S. Zhu, Frobenius and Separable Functors for Generalized Hopf Modules and Nonlinear Equations, Lecture Notes in Math. 1787, Springer, Berlin, 2002.

[10] H. Cartan and S. Eilenberg, Homological Algebra, Princeton Univ. Press, Princeton, 1956.

[11] M. Cipolla, Discesa fedelemente piatta dei moduli, Rend. Circ. Mat. Palermo (2) 25 (1976), 43-46.

[12] A. Connes, Non-commutative differential geometry, Inst. Hautes Études Sci. Publ. Math. 62 (1985), 257-360.

[13] J. Cuntz and D. Quillen, Algebra extensions and nonsingularity, J. Amer. Math. Soc. 8 (1995), 251-289.

[14] Y. Doi, On the structure of relative Hopf modules, Comm. Algebra 11 (1983), 243-253.

[15] Y. Dod, Unifying Hopf modules, J. Algebra 153 (1992), 373-385.

[16] L. El Kaoutit, J. Gómez-Torrecillas and F. J. Lobillo, Semisimple corings, preprint, 2001.

[17] J. Gómez-Torrecillas, Separable functors for corings, Int. J. Math. Math. Sci. 30 (2002), 203-226.

[18] F. Guzman, Cointegrations, relative cohomology for comodules and coseparable corings, J. Algebra 126 (1989), 211-224.

[19] M. Kleiner, The dual ring to a coring with a grouplike, Proc. Amer. Math. Soc. 91 (1984), $540-542$. 
[20] M. A. Knus and M. Ojanguren, Théorie de la descente et algèbres d'Azumaya, Lecture Notes in Math. 389. Springer, Berlin, 1974.

[21] M. Koppinen, Variations on the smash product with applications to group-graded rings, J. Pure Appl. Algebra 104 (1994), 61-80.

[22] M. Koppinen, On twistings of comodule algebras, Comm. Algebra 25 (1997), 2009-2027.

[23] P. Nuss, Noncommutative descent and non-Abelian cohomology, K-Theory 12 (1997), 2374.

[24] D. E. Radford and J. Towber, Yetter-Drinfeld categories associated to an arbitrary algebra, J. Pure Appl. Algebra 87 (1993), 259-279.

[25] A.V. Rojter, Matrix problems and representations of BOCS's, in: Lecture Notes in Math. 831, Springer, Berlin, 1980, 288-324.

[26] M. E. Sweedler, Groups of simple algebras, Inst. Hautes Études Sci. Publ. Math. 44 (1975), 79-189.

[27] M. E. Sweedler, The predual theorem to the Jacobson-Bourbaki theorem, Trans. Amer. Math. Soc. 213 (1975), 391-406.

[28] M. Takeuchi, A correspondence between Hopf ideals and sub-Hopf algebras, Manuscripta Math. 7 (1972), 251-270.

[29] R. Wisbauer, Weak corings, J. Algebra 245 (2001), 123-160.

[30] R. Wisbauer, On the category of comodules over corings, in: Mathematics \& Mathematics Education (Bethlehem, 2000), World Sci., River Edge, NJ, 2002, 325-336.

[31] D. N. Yetter, Quantum groups and representations of monoidal categories, Math. Proc. Camb. Phil. Soc. 108 (1990), 261-290.

[32] B. Zimmermann-Huisgen, Pure submodules of direct products of free modules, Math. Ann. 224 (1976), 233-245. 